\title{
Nonalcoholic Fatty Liver Disease and Fibrosis Risk in Patients With Obstructive Sleep Apnea: A Retrospective Analysis
}

\author{
Hani A. Jawa ${ }^{1}$, Hazim Khatib ${ }^{1}$, Naif Alzahrani ${ }^{1}$, Ahmed Alawi ${ }^{1}$, Mohanad AL-Gamdi ${ }^{1}$, Ahmed \\ Abuljadayel ${ }^{1}$, Sarah Altayyari ${ }^{1}$, Faris Alhejaili ${ }^{1}$, Mahmoud Mosli ${ }^{1}$, Siraj O. Wali ${ }^{1}$ \\ 1. Department of Medicine, King Abdulaziz University, Jeddah, SAU
}

Corresponding author: Sarah Altayyari, saraaltayyar@gmail.com

\section{Abstract \\ Objectives}

Obstructive sleep apnea (OSA) and nonalcoholic fatty liver disease (NAFLD) are prevalent and commonly associated conditions. We aimed to estimate the prevalence of NAFLD and identify liver fibrosis risk using noninvasive scoring methods in a cohort of patients with OSA.

\section{Methodology}

In this retrospective study of patients with confirmed OSA, patients who underwent abdominal ultrasonography were recruited. The primary outcome was the prevalence of suspected NAFLD (steatosis on ultrasound and/or elevated alanine transaminase [ALT]). The secondary outcomes included the prevalence and predictors of liver fibrosis risk as assessed by the NAFLD fibrosis score (NFS) and fibrosis-4 (FIB-4) score.

\section{Results}

A total of 133 patients fulfilled the study inclusion criteria. The average age was $49.8 \pm 15.1$ years, and $57.1 \%$ were females. The average BMI was $37.3 \pm 14.5$. According to the apnea-hypopnea index (AHI) scores, $37.9 \%$, $29.6 \%$, and $32.6 \%$ of participants had mild (5-14), moderate (15-29), and severe (>=30) OSA, respectively. Radiologically defined NAFLD was detected in $44.4 \%$ of the participants, and elevated liver enzymes were detected in $63.9 \%$ of the patients. High NFS and FIB- 4 scores were recorded in $9 \%$ and $3.8 \%$ of the patients, respectively. According to logistic regression analysis, age and BMI significantly predicted high NFS scores.

\section{Conclusion}

NAFLD appears to be common among OSA patients. Age and obesity, but not OSA severity, predicted high liver fibrosis risk as assessed by noninvasive scoring systems.

Review began 02/01/2021 Review ended 02/21/2021 Published 02/28/2021

\section{() Copyright 2021}

Jawa et al. This is an open access article distributed under the terms of the Creative Commons Attribution License CC-BY 4.0., which permits unrestricted use, distribution, and reproduction in any medium, provided the original author and source are credited.
Categories: Internal Medicine, Gastroenterology, Pulmonology

Keywords: fatty liver disease, obstructive sleep apnoea, liver fibrosis

\section{Introduction}

Obstructive sleep apnea (OSA) and nonalcoholic fatty liver disease (NAFLD) are highly prevalent medical conditions, and both are commonly associated with obesity and metabolic syndrome. Globally, the prevalence of NAFLD is estimated to be approximately $25 \%$, whereas the prevalence of OSA in Saudi Arabia is $8.5 \%$ (12.8\% in males and $4.8 \%$ in females) [1-3]. Over the past few years, accumulating evidence has shown OSA to be independently related to the development and progression of NAFLD [4-7]. The mechanisms behind this association are not well identified, but chronic intermittent hypoxia (CIH), as quantified by oxygen saturation, has been shown to be an important trigger [8]. In fact, one study demonstrated that in the absence of obesity, CIH leads to mild liver injury via oxidative stress and excessive glycogen accumulation in hepatocytes, thus sensitizing the liver to a second insult [9].

Liver fibrosis is an established independent predictor of disease-specific and overall mortality in patients with NAFLD [10]. Therefore, early identification and staging of fibrosis have become increasingly important, especially in patients with risk factors for progression from simple steatosis to advanced fibrosis and cirrhosis [11]. Histopathological examination of a liver biopsy is considered the gold standard for staging liver fibrosis [12]. However, it is invasive in nature and carries a small yet potentially serious risk of complications and therefore should be performed only when the expected benefits outweigh the risks, especially in the context of a highly prevalent condition such as NAFLD. Therefore, simple and noninvasive testing using clinical decision aids has been developed and utilized to predict fibrosis risk in patients with NAFLD. Of these tests, two of the most validated are the NAFLD fibrosis score (NFS) and fibrosis-4 (FIB-4) [12]. These scores are calculated using readily available patient demographic data and biochemical tests and 
can detect advanced fibrosis with modest accuracy when used alone and can reliably exclude advanced fibrosis in a high proportion of patients with NAFLD [11].

The aim of this study was to estimate the prevalence of suspected NAFLD and perform risk stratification of fibrosis among OSA patients using noninvasive scores. Additionally, we aimed to identify possible predictors of liver steatosis and fibrosis in this patient population.

\section{Materials And Methods}

We performed a retrospective study of consecutive adult patients evaluated at a Sleep Medicine and Research Center between January 2016 and August 2018. We included all patients with confirmed OSA based on full polysomnography who underwent abdominal ultrasonography for various indications. The following data were collected: (A) Demographics including age, sex, height, weight and body mass index; (B) Clinical parameters including history of metabolic risk factors such as diabetes, hypertension and hyperlipidemia; (C) Laboratory data including complete blood counts, liver enzymes, lipid profile, and hemoglobin A1C; (D) Radiological tests including findings of steatosis or cirrhosis seen on abdominal ultrasound (U/S); and (E) Polysomnography data including the apnea-hypopnea index (AHI). Patients with a history of viral hepatitis or chronic liver disease other than fatty liver and patients with any documented alcohol consumption were excluded.

After completing data collection, the FIB-4 index and NFS were calculated using their respective validated equations as follows:

(A) NFS $=\left(-1.675+0.037 \times\right.$ age $($ years $)+0.094 \times$ BMI $\left(\mathrm{kg} / \mathrm{m}^{2}\right)+1.13 \times \mathrm{IFG} /$ diabetes $($ yes $=1$, no $=0)+0.99 \times$ AST/ALT ratio $-0.013 \times$ platelets $\left(\times 10^{9} / \mathrm{L}\right)-0.66 \times$ albumin $\left.(\mathrm{g} / \mathrm{dl})\right)$.

(B) FIB-4 = (age $x$ AST) $/$ (platelets $x$ sqr ALT).

\section{Definitions and outcomes}

Suspected NAFLD was defined as liver steatosis on abdominal US and/or elevated liver enzymes in patients with risk factors. Elevated ALT was defined as $>30$ IU/L for males and >19 IU/L for females. Liver fibrosis risk was categorized into low risk (NFS <=1.455 and FIB-4 <1.30) and high risk (NFS>0.676 and FIB-4>2.67).

The diagnosis of OSA was made based on the American Academy of Sleep Medicine (AASM) diagnostic criteria [13]. Furthermore, OSA severity was categorized as mild (AHI=5-15), moderate (AHI=15-30), and severe $(\mathrm{AHI}=>30$ ) based on the AASM recommendations.

The primary outcome of the study was the prevalence of suspected NAFLD among patients with OSA. Secondary outcomes included the prevalence of high and low liver fibrosis risk scores, US-diagnosed liver cirrhosis, factors predictive of NAFLD and high NFS and FIB-4 scores.

\section{Statistical analysis}

Descriptive statistics were calculated for baseline characteristics; means and standard deviations (SD) are used to describe continuous variables, and frequencies are used to describe categorical variables.

Accordingly, a standard Student's t-test was used to compare means, and chi-square was used to compare frequencies. Logistic regression analysis was used to study associations with

binary dependent variables. Odds ratios (ORs) and 95\% confidence intervals (CIs) were estimated. STATA

11.2 (StataCorp, College Station, TX, USA) was used in our analysis. Statistical significance was set at 0.05.

\section{Ethical considerations}

This study was approved by the Hospital's Institutional Review Board (IRB) of (Reference No 378-18).

\section{Results}

\section{Baseline characteristics}

A total of 133 patients fulfilled the study inclusion criteria (Table 1). 


\section{Cureus}

\begin{tabular}{|c|c|c|}
\hline \multirow[t]{2}{*}{ Characteristic } & \multicolumn{2}{|l|}{ Summary statistics } \\
\hline & NAFLD on US ( $n=59)$ & No NAFLD on US $(n=74)$ \\
\hline \multicolumn{3}{|l|}{ Demographics } \\
\hline Mean age (SD), years & $49.7(1.7)$ & $49.8(1.9)$ \\
\hline Mean BMI (SD), kg/m² & $38.9(2.4)$ & 36.0 (1.2) \\
\hline Female sex & 35 & 41 \\
\hline \multicolumn{3}{|l|}{ Comorbiditles } \\
\hline Diabetes mellitus & 23 & 20 \\
\hline Dyslipidemia & 7 & 10 \\
\hline Hypertension & 23 & 27 \\
\hline Liver cirrhosis & 1 & 3 \\
\hline \multicolumn{3}{|l|}{ OSA severity } \\
\hline Mild (AHI = 5-15) & 21 & 29 \\
\hline Moderate (AHI = 15-30) & 12 & 27 \\
\hline Severe (AHI => 30) & 26 & 17 \\
\hline \multicolumn{3}{|l|}{ Suspected NAFLD } \\
\hline Elevated ALT & 42 & 43 \\
\hline \multicolumn{3}{|l|}{ Liver fibrosis scores } \\
\hline High FIB-4 & 1 & 4 \\
\hline High NFS & 5 & 7 \\
\hline Low FIB-4 & $54 / 59$ & $62 / 74$ \\
\hline Low NFS & 33 & 38 \\
\hline
\end{tabular}

\section{TABLE 1: Baseline characteristics of 133 patients with confirmed OSA.}

OSA: obstructive sleep apnea; AHI: apnea-hypopnea index; US: ultrasound; NAFLD: nonalcoholic fatty liver disease; ALT: alanine transaminase; FIB4: fibrosis-4 score; NFS: NAFLD fibrosis score.

The average age was $49.8 \pm 15.1$, and $57.1 \%$ were females; $37.6 \%, 32.3 \%$ and $12.8 \%$ had hypertension, diabetes, and dyslipidemia, respectively. The average BMI was $37.3 \pm 14.5$. The average serum albumin concentration, AST and ALT were $35.4( \pm 4.1), 26.4( \pm 39.9)$, and $39.5( \pm 74.3)$, respectively. The average platelets were 291 ( \pm 10.6 .2 ). According to AHI scores, $37.9 \%, 29.6 \%$, and $32.6 \%$ had mild (5-14), moderate (15-29), and severe (>=30) OSA, respectively.

\section{Outcomes}

Radiologically suspected NAFLD was detected in $44.4 \%$ of participants, biochemically suspected NAFLD (elevated ALT liver enzymes) was detected in $63.9 \%$ of participants, and features consistent with liver cirrhosis were found in $3 \%$ of this cohort. High NFS and FIB-4 scores were recorded in $9 \%$ and $3.8 \%$ of all OSA patients, respectively. Low NFS and FIB-4 scores were recorded in $53 \%$ and $87 \%$ of all OSA patients, respectively.

\section{Predictors of fibrosis}

According to logistic regression analysis, age $(\mathrm{OR}=1.1, \mathrm{p}=0.018)$, liver cirrhosis $(\mathrm{OR}=1326, \mathrm{p}=0.008)$, and $\mathrm{BMI}$ $(\mathrm{OR}=1.2, \mathrm{p}=0.009)$ significantly predicted high NFS scores, and liver cirrhosis $(\mathrm{OR}=62.5, \mathrm{p}=0.047)$ predicted high FIB-4 scores (Table 2). 


\section{Cureus}

\begin{tabular}{|c|c|c|c|c|}
\hline Hight NFS & Odds ratio & $P$ & $95 \%$ confidence interval & $95 \%$ confidence interval \\
\hline Age & 1.124764 & 0.018 & 1.019999 & 1.240288 \\
\hline Sex & 1.190144 & 0.879 & $0.12 / 6972$ & 11.09219 \\
\hline AHI severity & 1.024474 & 0.186 & 0.9883832 & 1.061882 \\
\hline BMI & 1.163037 & 0.009 & 1.0382 & 1.302885 \\
\hline Liver cirrhosis & 1326.183 & 0.008 & 6.335305 & 277612.7 \\
\hline Elevated ALT & 0.8622967 & 0.895 & 0.0958865 & 7.754543 \\
\hline Diabetes & $2.2697 / 9$ & 0.459 & 0.2598809 & 19.82407 \\
\hline Dyslipidemia & 0.2402186 & 0.456 & 0.0056482 & 10.21647 \\
\hline Hypertension & 0.5578992 & 0.615 & 0.0573876 & 5.42367 \\
\hline High FlB-4 & Odds ratio & $P$ & $95 \%$ confidence interval & $95 \%$ confidence interval \\
\hline Age & 1.03609 & 0.449 & 0.9451864 & 1.135736 \\
\hline Sex & 0.8935471 & 0.0923 & 0.0909076 & 8.782836 \\
\hline BMI & 0.9789711 & 0.715 & 0.8734246 & 1.097272 \\
\hline AHI severity & $0.9861 / / 8$ & 0.584 & 0.938203 & 1.036606 \\
\hline Diabetes & 0.0219631 & 0.025 & 0.000781 & 0.617656 \\
\hline Dysilipidemia & 10.01055 & 0.177 & 0.3530267 & 283.8625 \\
\hline Hypertension & 2.642495 & 0.419 & 0.2498476 & 27.94816 \\
\hline
\end{tabular}

TABLE 2: Predictors of high fibrosis risk as assessed by noninvasive scores.

BMI: body mass index; AHI: apnea-hypopnea index; ALT: alanine transaminase; FIB-4: fibrosis-4 score; NFS: NAFLD fibrosis score.

However, no significant predictors of radiologically suspected fatty liver could be identified statistically (Table 3).

\begin{tabular}{|c|c|c|c|c|}
\hline Fatty liver & Odds ratio & $\mathbf{P}$ & $95 \%$ confidence Interval & $95 \%$ confidence interval \\
\hline Age & 1.000331 & 0.981 & 0.9729921 & 1.028438 \\
\hline Sex & 1.561487 & 0.275 & 0.7010942 & 3.477764 \\
\hline $\mathrm{AHI}$ & 1.01511 & 0.057 & 0.9995392 & 1.030924 \\
\hline BMI & 1.014762 & 0.275 & 0.9883864 & 1.041841 \\
\hline Diabetes & 2.25053 & 1.71 & 0.887361 & 5.707806 \\
\hline Dyslipidemia & .4374361 & 0.200 & 0.1234384 & 1.550169 \\
\hline Hypertension & .7770351 & 0.609 & 0.2951852 & 2.04544 \\
\hline Elevated ALT & 1.560633 & 0.277 & 0.7000332 & 3.479228 \\
\hline
\end{tabular}

TABLE 3: Predictors of steatosis as assessed by abdominal US.

ALT: alanine transaminase; US: ultrasound; AHI: apnea-hypopnea index; BMI: body mass index.

\section{Discussion}


In this retrospective study, 133 patients had confirmed OSA and underwent abdominal ultrasonography. Radiologically defined NAFLD was detected in $44.4 \%$ of patients, and elevated liver enzymes were detected in $63.9 \%$ of patients. High NFS and FIB-4 scores were recorded in $9 \%$ and $3.8 \%$ of patients with OSA, respectively. Age and BMI were found to significantly predict high NFS scores. Since the establishment of liver fibrosis as a strong predictor of liver-related outcomes and mortality in patients with NAFLD, there has been increasing interest in the development and application of noninvasive, readily available and costefficient tests to detect liver fibrosis in patients with this highly prevalent medical condition. Of these tests, FIB-4 and NFS have been recommended for use by practice guidelines for the initial evaluation of patients with NAFLD, especially those at higher risk of nonalcoholic steatohepatitis (NASH), which is the progressive form of NAFLD that can lead to fibrosis and cirrhosis in a subset of patients [11,14]. Patients who are considered to be at a higher risk for NASH include those with advanced age, type 2 diabetes and other components of the metabolic syndrome [12]. No current recommendations, however, are in place regarding whether to routinely screen OSA patients for NAFLD and fibrosis irrespective of the abovementioned NASH risk factors. A recent meta-analysis of 9 studies that included 2272 participants with OSA who underwent bariatric surgery and liver biopsy found OSA and its degree of severity to be independently linked to ALT levels and found OSA to correlate with histological features of NAFLD including fibrosis but not with the NAFLD activity score [6].

The proven association between OSA and NAFLD, irrespective of other risk factors, can be an argument for NAFLD and fibrosis screening in this patient population, especially those with severe sleep apnea. In our study, among 133 patients, most were obese, at least one-third of them had a component of metabolic syndrome, and suspicion of NAFLD was common. Ultrasound-detected steatosis was found in $44.4 \%$ of patients. This estimate is considered higher than the prevalence of fatty liver in the general population (approximately 25\%) and comparable to that in studies reporting liver biopsy data from bariatric surgery patients with OSA in which steatosis with or without inflammation was found in 44 to $84 \%$ of cases [7]. Notably, the sensitivity of US for diagnosing fatty liver is reduced when there is $<30 \%$ steatosis and in patients with morbid obesity, which is common in OSA patients [15].

Additionally, we identified $63.9 \%$ of cases with elevated ALT using the revisited sex-specific cutoff levels, which may be more sensitive in detecting NAFLD [16]. This is comparable to a recent large French study that assessed the association between OSA and blood markers of liver injury; the study reported elevated ALT in $53 \%$ of patients with severe OSA. With regard to the secondary outcomes of this study, the prevalence of low fibrosis risk assessment was different between the two applied scores: $53 \%$ using NFS and $87 \%$ using FIB-4, which may be due to differences in test performance or the effect of other variables such as patient age. The remaining patients with intermediate or high fibrosis risk would require referral to a hepatology clinic for further assessment by an additional noninvasive tool, namely, elastography or a liver biopsy.

Regarding predictors of fibrosis, we could identify only age and BMI as predictors of high NFS. Both factors are linked to the risk of NASH and fibrosis in NAFLD patients. Expectedly, the finding of liver cirrhosis on US was related to high NFS and FIB-4 scores. With regard to OSA severity, a predictive role for high fibrosis scores was not observed in our cohort, and the results of this association conflict with those of other larger studies $[7,17]$.

Although this study was limited by its retrospective nature, sample size and lack of liver biopsy data, it highlights the magnitude of the prevalence of NAFLD among OSA patients and demonstrates the applicability of NFS and FIB-4 scores as first-line tools for fibrosis risk assessment.

\section{Conclusions}

NAFLD commonly occurs in patients with OSA. Application of the NFS and FIB-4 tools to assess liver fibrosis risk would circumvent the need for liver biopsy in many patients who require careful monitoring and appropriate management, including weight loss, treating metabolic risk factors and continuous positive airway pressure (CPAP) treatment when indicated.

\section{Additional Information \\ Disclosures}

Human subjects: Consent was obtained or waived by all participants in this study. Institutional Review Board at King Abdulaziz University issued approval (Reference No 378-18). This study was approved by the Institutional Review Board at King Abdulaziz University (Reference No 378-18). Animal subjects: All authors have confirmed that this study did not involve animal subjects or tissue. Conflicts of interest: In compliance with the ICMJE uniform disclosure form, all authors declare the following: Payment/services info: All authors have declared that no financial support was received from any organization for the submitted work. Financial relationships: All authors have declared that they have no financial relationships at present or within the previous three years with any organizations that might have an interest in the submitted work. Other relationships: All authors have declared that there are no other relationships or activities that could appear to have influenced the submitted work. 


\section{References}

1. Younossi Z, Anstee QM, Marietti M, et al.: Global burden of NAFLD and NASH: trends, predictions, risk factors and prevention. Nat Rev Gastroenterol Hepatol. 2018, 15:11-20. 10.1038/nrgastro.2017.109

2. Senaratna CV, Perret JL, Lodge CJ, et al.: Prevalence of obstructive sleep apnea in the general population: a systematic review. Sleep Med Rev. 2017, 34:70-81. 10.1016/j.smrv.2016.07.002

3. Wali SO, Abalkhail B, Krayem A: Prevalence and risk factors of obstructive sleep apnea syndrome in a Saudi Arabian population. Ann Thorac Med. 2017, 12:88-94. 10.4103/1817-1737.203746

4. Benotti P, Wood GC, Argyropoulos G, et al.: The impact of obstructive sleep apnea on nonalcoholic fatty liver disease in patients with severe obesity. Obesity. 2016, 24:871-877. 10.1002/oby.21409

5. Chou TC, Liang WM, Wang CB, et al.: Obstructive sleep apnea is associated with liver disease: a populationbased cohort study. Sleep Med. 2015, 16:955-960. 10.1016/j.sleep.2015.02.542

6. Jin S, Jiang S, Hu A: Association between obstructive sleep apnea and non-alcoholic fatty liver disease: a systematic review and meta-analysis. Sleep Breathing. 2018, 22:841-851. 10.1007/s11325-018-1625-7

7. Weingarten TN, Mantilla CB, Swain JM, et al.: Nonalcoholic steatohepatitis in bariatric patients with a diagnosis of obstructive sleep apnea. Obes Facts. 2012, 5:587-596. 10.1159/000342677

8. Aron-Wisnewsky J, Minville C, Tordjman J, et al.: Chronic intermittent hypoxia is a major trigger for nonalcoholic fatty liver disease in morbid obese. J Hepatol. 2012, 56:225-233. 10.1016/j.jhep.2011.04.022

9. Savransky V, Nanayakkara A, Vivero A, et al.: Chronic intermittent hypoxia predisposes to liver injury . Hepatology. 2007, 45:1007-1013. 10.1002/hep.21593

10. Ekstedt M, Hagstrom $\mathrm{H}$, Nasr P, et al.: Fibrosis stage is the strongest predictor for disease-specific mortality in NAFLD after up to 33 years of follow-up. Hepatology. 2015, 61:1547-1554. 10.1002/hep.27368

11. EASL-EASD-EASO: Clinical Practice Guidelines for the management of non-alcoholic fatty liver disease . J Hepatol. 2016, 64:1388-1402. 10.1016/j.jhep.2015.11.004

12. Tsai E, Lee TP: Diagnosis and evaluation of nonalcoholic fatty liver disease/nonalcoholic steatohepatitis, including noninvasive biomarkers and transient elastography. Clin Liver Dis. 2018, 22:73-92. 10.1016/j.cld.2017.08.004

13. Kapur VK, Auckley DH, Chowdhuri S, et al.: Clinical practice guideline for diagnostic testing for adult obstructive sleep apnea: An American Academy of Sleep Medicine Clinical Practice Guideline. J Clin Sleep Med. 2017, 13:479-504. 10.5664/jcsm.6506

14. Chalasani N, Younossi Z, Lavine JE, et al.: The diagnosis and management of nonalcoholic fatty liver disease: practice guidance from the American Association for the Study of Liver Diseases. Hepatology. 2018, 67:328-57. 10.1002/hep.29367

15. Saadeh S, Younossi ZM, Remer EM, et al.: The utility of radiological imaging in nonalcoholic fatty liver disease. Gastroenterology. 2002, 123:745-750. 10.1053/gast.2002.35354

16. Prati D, Taioli E, Zanella A, et al.: Updated definitions of healthy ranges for serum alanine aminotransferase levels. Ann Intern Medicine. 2002, 137:1-10. 10.7326/0003-4819-137-1-200207020-00006

17. Trzepizur W, Boursier J, Mansour Y, et al.: Association between severity of obstructive sleep apnea and blood markers of liver injury. Clin Gastroenterol Hepatol. 2016, 14:1657-1661. 10.1016/j.cgh.2016.04.037 